\title{
Central Nervous System Lesions in COVID-19
}

\author{
O. V. Kurushina and A. E. Barulin
}

Translated from Zhurnal Nevrologii i Psikhiatrii imeni S. S. Korsakova, Vol. 121, No. 1, Iss. 1, pp. 92-97, January, 2021. Original article submitted December 2, 2020. Accepted January 12, 2021.

This review discusses current data on CNS lesions in infections with the new coronavirus that causes COVID-19. The pathogenetic mechanisms leading to infection of the brain and spinal cord are presented. Published studies and meta-analyses published by autumn 2020 are assessed. The authors present results from their own clinical observations of various types of CNS infection in COVID-19 virus disease. Particular attention is paid to certain common forms of CNS lesion such as encephalitis, cerebrovascular pathology, and headache. A form of acute hemorrhagic necrotic encephalopathy is identified, which is a quite rare but fatal pathology comorbid with COVID-19. A description of our own clinical observation of acute necrotic encephalopathy is presented. The importance of further studies of the effects of the coronavirus on the CNS and possible approaches to treatment and rehabilitation of this category of patients is emphasized.

Keywords: COVID-19, acute hemorrhagic necrotic encephalopathy, central nervous system.

In March 2020, the WHO declared a pandemic caused by the COVID-19 virus. From this point in time it became evident that the population had encountered a novel threat with unknown outcomes and sequelae despite the fact that this type of virus is known to investigators and had already manifested a number of spikes of severe acute respiratory syndrome (SARS) and middle east respiratory syndrome (MERS) in 2002-2003 and 2012. The new variant, SARSCoV-2, was first detected in Wuhan, China, in November 2019 [1-3]. Even at the beginning of the pandemic, it became apparent to investigators that despite the name of the respiratory syndrome, infection with the novel type of coronavirus was not limited to the respiratory system but is in fact a multiorgan and multisystem infection. Central nervous system lesions are not the first manifesting symptoms, but often account for the severity of the patient's condition, the long-term sequelae, and sometimes lethal outcomes.

Studies indicate that more than $35 \%$ of patients with COVID-19 develop neurological symptoms [4,5]. However, there are as yet no systematic data on the distribution and spectrum of neurological manifestations in the new coronavirus infection. Some studies have emphasized that the prevalence of neurological signs and symptoms is greater in

Volgograd State Medical University, Russian Ministry of Health, Volgograd, Russia; e-mail: ovkurushina@mail.ru. patients with severe COVID-19 infection, which may be the result of cerebral hypoxia due to respiratory failure [6,7]. However, the pathogenesis of neurological symptoms in this diseases may not be limited to this mechanism.

A whole series of pathogenetic variants have now been proposed to explain the possible involvement of SARS$\mathrm{CoV}-2$ in the nervous system, including direct invasion of the virus through impairments to the blood-brain barrier (BBB) due to release of cytokines and retro- and anterograde neuronal transport involving dynein and kinesin proteins [8]. In addition, possible nerve damage may be mediated by the immune system, hypoxia due to pneumonia, and/or binding to angiotensin-converting enzyme 2 (ACE2) receptors [9-11]. Studies indicate that the surface spike glycoprotein of SARSCoV-2 may interact with ACE2 receptors, leading to endothelial dysfunction and secondary neurological impairments due to damage to the microcirculation. Steardo et al. showed that coronavirus has a direct damaging action on the brainstem neurons responsible for cardiorespiratory regulation, which leads to respiratory failure and hypoxia [12].

On the one hand, SARS-CoV-2 appears to lead to both pneumonia and dysfunction of the brainstem cardiorespiratory center, exacerbating pneumonia. Severe pneumonia can lead to systemic hypoxia, producing brain damage. Hypoxia arising as a result of severe respiratory distress syndrome and pneumonia due to COVID-19 primarily af- 
fects the brain via various physiological and compensatory changes leading to neurological manifestations and pathology [13]. Hypoxia leads to metabolic acidosis, intracellular accumulation of lactic acid, increases in the quantity of free radicals, and decreases in ATP production [14]. Decreased blood saturation induces dilation of the intracranial vascular bed and neuron membrane damage, promoting neuron swelling, interstitial cerebral edema, and ultimately the development of irreversible damage [15].

On the other hand, hypoxia can exacerbate CNS damage, leading to the formation of a vicious circle resulting in lethal outcome. This supports the wider distribution of neurological symptoms in severe patients [16].

Other mechanisms for the development of CNS damage must also be considered, particularly immune and autoimmune lesions. Immunologically mediated damage occurring mainly because of cytokine storms, with increases in the levels of inflammatory cytokines and activation of the T-lymphocytes, macrophages, and endothelial cells. Further interleukin-6 release induces excessive leakage from blood vessels, activation of the complement and coagulation cascades, disseminated intravascular coagulation, and damage to target organs, including the brain and spinal cord $[17,18]$.

Considering the lack of evidence and the importance of the possible role of CNS and peripheral nervous system damage in the clinical picture and outcomes of the new coronavirus infection, further research in this direction is needed.

Clinical Forms of Nervous System Damage. A retrospective series of cases from January 16, 2020 to February 18,2020 in Wuhan, China, showed that $36.4 \%$ of patients showed neurological symptoms, of which $24.8 \%$ were CNS lesions and $8.9 \%$ were peripheral lesions. The incidence of neurological symptoms correlated with the severity of respiratory dysfunction on the background of SARS-CoV-2 infection, and neurological symptoms were seen more frequently in elderly patients, in whom comorbid diseases, particularly arterial hypertension, were more common [19].

Another group of investigators ran a single-center retrospective analysis of all COVID-19 patients presenting to one of the repurposed departments of intensive respiratory therapy at an academic center in New Orleans (Louisiana, USA) on April 22, 2020. A total of 76 patients were hospitalized, of whom 27 patients (35.5\%) had signs of new neurological disease, which varied from mild headache and dysgeusia to severe focal neurological deficit. All but one patient with neurological manifestations displayed changes in psychoemotional status during admission (96.3\%) [20].

It must be noted that attempts have been made to produce systematic reviews and meta-analyses of almost 4700 patients from more than 41 published articles on the neurological manifestations of COVID-19. Common symptoms included asthenia (33.2\%), anorexia $(30.0 \%)$, malaise (26.7\%), breathlessness $(26.9 \%)$, myalgia $(16.0 \%)$, vertigo (10.0\%), headache (9.2\%), depressed consciousness (5.2\%), and nausea and vomiting (5.1\%) [21].
A number of investigators in different countries believe that the commonest signs of CNS damage are acute hemorrhagic encephalopathy, encephalitis, cerebrovascular disease, headache, and vertigo [22].

Acute Hemorrhagic Necrotic Encephalopathy. Acute hemorrhagic necrotic encephalopathy (AHNE) is a rare progressive neurodegenerative disease characterized by multiple symmetrical areas of edema and necrosis in the CNS developing during or after virus infection. AHNE has previously been described as a complication of viral invasion, particularly with influenza virus, and is most commonly found in children. To date only occasional cases of this encephalopathy have been described in adults. The main damaging factor in AHNE is believed to be a cytokine storm, which leads to disruption of the BBB without direct viral invasion [23, 24]. The clinical signs of AHNE are nonspecific, changes in mental state being the most common, particularly quantitative and qualitative impairments to consciousness (from suppression to delirium and coma) and convulsions. Virus is not found in the cerebrospinal fluid (CSF) or brain matter and analysis of CSF shows no significant changes. Endothelial damage to brain vessels is detected microscopically, confirming the view that cytokine damage to the BBB occurs, the most typical feature being symmetrical foci of petechial hemorrhages and necrosis in the brain matter, with areas of edema around the foci. These changes are seen on neuroimaging on CT and MRI scans and provide grounds for confirming diagnoses of AHNE.

The first report of the possible occurrence of AHNE on the background of SARS-CoV-2 infection appeared in spring 2020 , when a case history of a female patient aged 50 years was published. The initial signs included fever, cough, and changes in mental status; results from tests for influenza virus were negative, while nasopharyngeal smears were positive for SARS-CoV-2, confirming the diagnosis. Brain CT and MRI imaging demonstrated symmetrical hemorrhagic foci in the medial parts of the thalamus and temporal lobes [25].

We observed patient S., age 20 years, who, on a background of extensive tuberculosis infection of the lungs, was confirmed to have additional infection with SARS-CoV-2 virus. Neurological symptoms arose in the first week of illness, the first being mild changes in psychoemotional state, including anxiety, mild cognitive deficiency, insomnia, and nightmares. Considering the severity of the somatic illness, these symptoms were detected retrospectively. Neurological examination was conducted in the second week of the infectious disease, when the patient developed a tonic-clonic seizure without loss of consciousness in the evening, without any obvious cause. Neurological assessment showed no focal neurological pathology; standard electroencephalography performed outside the seizure period revealed no changes. The patient was given antiepileptic therapy though, despite high drug doses, brief seizures continued and cognitive impairments increased. The patient underwent an MRI scan, which demonstrated pathological increases in signal 


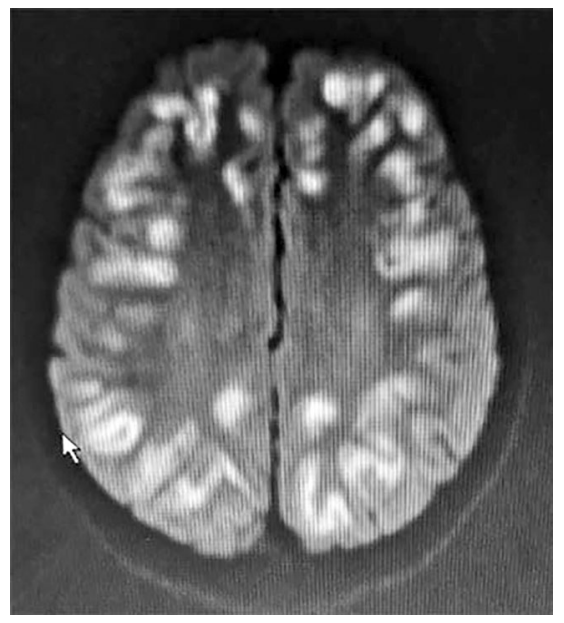

Fig. 1. MRI scan of patient $\mathrm{S}$ with a diagnosis of AHNE.

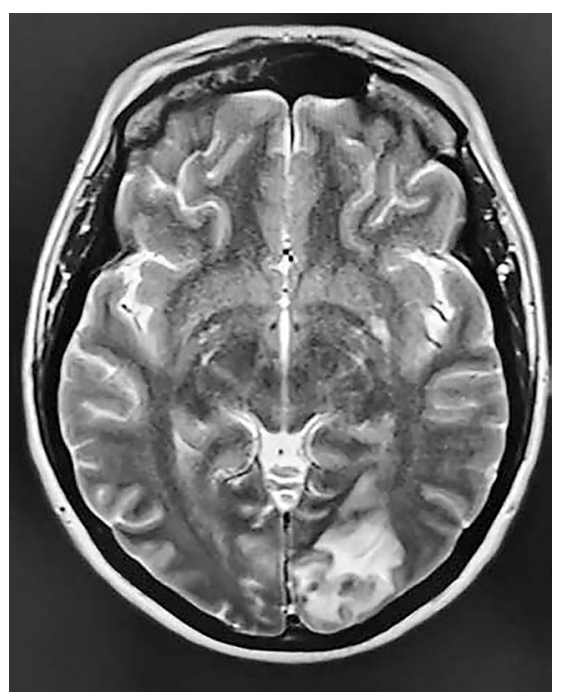

Fig. 2. MRI scan of patient Kh with a diagnosis of "encephalitis on the background of infection with SARS-CoV-2."

intensity on T2/TIRM and DWI from the cortical matter of the brain, with symmetrical changes in signal from the basal ganglia with hemorrhagic transformation, symmetrical increases in signal from the medial parts of the thalamus and periaqueductal gray matter on T2-TIRM images (Fig. 1). Patient $\mathrm{S}$ was diagnosed with AHNE on the basis of the neuroimaging data and the clinical picture and was prescribed methylprednisolone. However, the patient's impairment to consciousness increased despite treatment, and after a few days severe multiorgan failure led to death.

Encephalitis on the Background of SARS-CoV-2 Infection. Symptoms of encephalitis in SARS-CoV-2 infection include fever, headache, convulsions, behavioral disorders, and changes in the level of consciousness. Early diagnosis is a key factor in decreasing mortality in this category of patients, as the symptoms may initially be masked by the clinical picture of pneumonia and severe hypoxia. A case of encephalitis in a 56-year-old woman from Wuhan, who was diagnosed with COVID-19 in January 2020, was described [26]. A second case of meningoencephalitis was described in a 24-year-old Japanese with symptoms of COVID-19 who showed generalized convulsions and impairment of consciousness. CSF analysis revealed minor pleocytosis at 12 cells/ $\mu$ l. A brain MRI scan revealed hyperintense areas in the right periventricular area, the mesial area of the temporal lobe, and hippocampus. The patient required mechanical ventilation of the lungs because of pneumonia and repeated generalized convulsions [27].

In our practice, the case of patient $\mathrm{Kh}$ (59 years old, female) was indicative. This patient was admitted to a specialist hospital where on the background of confirmed pneumonia due to the new coronavirus infection, headache, vertigo, severe general weakness, distractibility of attention, and sleep disturbances arose in the second week of illness. The decision was made to transport the patient to the urgent care hospital or neuroimaging and neurology consultation. On examination, the neurological status of patient $\mathrm{Kh}$ included mild anisoreflexia, widespread muscle weakness with impairments to coordination, and mild lethargy. Neuroimaging results revealed foci of unilateral signal enhancement, confirming the diagnosis of encephalitis (Fig. 2). CSF examination revealed an increase in pressure to $220 \mathrm{~mm}$ water, lymphocytic pleocytosis (18 cells $/ \mu \mathrm{l})$, and no growth of microorganisms on culture. PCR investigations for viruses was not performed for technical reasons. The patient is currently receiving treatment in the neurology hospital.

Cerebrovascular Disease. Investigations of patients with COVID-19 in Italy showed that despite admission of patients with confirmed infection and prophylaxis against venous thromboembolism, the incidence of ischemic stroke increased by $2.5 \%$ [28]. Furthermore, patients with COVID-19 hospitalized in an intensive care department with confirmed infection in China showed an increase in the incidence of ischemic stroke by $5 \%$, while a study in the Netherlands showed an increase by 3.7\% [29]. In addition, signs of ischemic stroke were seen in younger patients infected with COVID-19. Finally, any comorbid disease in elderly patients, such as infection and the state of hypercoagulation, can increase the risk of ischemic stroke [30].

The fact that SARS-CoV-2 binds to ACE2 receptors on endothelial cells, inducing a massive inflammatory response, which increases blood vessel constriction, has been actively discussed in relation to acute circulatory compromise in target organs. The resultant process of inflammatory changes can lead to vasculitis or thrombosis, and thus to cerebral ischemia, as well as to altered BBB permeability, which may be the basis of intracerebral hemorrhages. The widespread introduction into the clinical recommendations of drugs preventing excessive thrombus formation emphasizes the importance of preventing the thrombotic complications of the new coronavirus infection. Another mechanism for the exacerbating or initiating cerebrovascular pathology has also been discussed [31]. Decreased expression 
of ACE receptors can evidently have adverse effects on the renin-angiotensin system, complicating control of vascular tone in the brain and spinal cord system and affecting the regulation of arterial blood pressure overall, which can potentially lead to acute cerebrovascular accidents $[32,33]$.

Unfortunately, the real influence of the new virus infection SARS-CoV-2 on the course of existing cerebrovascular pathology and the occurrence of new cerebrovascular lesions cannot be evaluated fully. On the background of a constant increase in the incidence of the disease in the current world, due to features including widespread risk factors and comorbid pathologies, it is not possible to identify or, in particular, confirm the role of virus invasion. Nonetheless, the fact that SARS-CoV-2 has influences on the circulatory system as a whole and on increases in cerebrovascular pathology and its long-term sequelae is beyond discussion.

Acute Transverse Myelitis. The proposed pathogenesis of acute transverse myelitis associated with SARS-CoV-2 infection is probably linked with the cytokine storm mechanism [34]; this induces an inflammatory reaction with release of macrophages, interleukins, interferons, and chemokines [34-36].

Munz et al. [36] reported acute transverse myelitis associated with COVID-19. A 60-year-old patient presenting with respiratory illness confirmed as SARS-CoV-2 by a positive RT-PCR test on an oropharyngeal smear was described, in which the patient received support therapy. Progressive neurological deficit appeared three days after discharge from hospital and included urinary bladder dysfunction and bilateral lower limb weakness. After a few days the symptoms progressed to include the upper limbs and signs of central motoneuron involvement appeared. Neuroimaging of the spinal cord yielded data confirming acute transverse myelitis. In this report the authors noted that the course of illness was notably improved after administration of methylprednisolone [37].

We observed patient A (22 years old, female) who attended the clinic complaining of acute urinary retention and increasing lower limb weakness. Two weeks previously the patient had fallen ill with pulmonary SARS-CoV-2 disease but had not sought medical advice. After recovery, the patient developed acute urinary retention assessed by a local urologist as a functional impairment in an emotionally labile young woman. Nonetheless, the treatment provided did not produce the desired effect, and after three days of deterioration in the patient's condition, with daily catheterization and mild weakness in the limbs, she was referred to the department of neurology. Neurological status at assessment of the patient showed: lower peripheral paraparesis, absent Achilles reflexes, sharply reduced patellar reflexes, impairment to deep and superficial sensation to the T6/7 level, and the absence of the sensation of bladder fullness. The patient had been catheterized the day before with positioning of a permanent catheter. During hospitalization, the patient's condition deteriorated over the first three days despite treat- ment with methylprednisolone, with increasing weakness in the legs to the level of plegia, anesthesia of superficial sensation in the distal areas, and lack of sensations from the pelvic organs. This was paralleled by increases in psychoemotional disturbance: severe emotional lability appeared, with tearfulness and insomnia. A spinal cord MRI scan demonstrated a focus of inflammatory change, hyperintense, at the T4-T8 level in T2-weighted mode. On contrasting, a focus of uneven contrast accumulation was seen. Analysis for aquaporin-4 antigen was negative. Patient A received hospital treatment for three weeks, with gradual improvement. She was discharged with residual signs in the form of mild hypesthesia and decreased muscle strength in the distal parts of the legs to 4 points. Out-patient treatment continues.

Headache and Vertigo. These symptoms are believed to be the commonest among all the neurological manifestations of the new coronavirus infection. A meta-analysis based on the clinical, laboratory, and imaging characteristics of COVID-19 from January 1, 2020 to February 23, 2020 showed that headache is the dominant CNS symptom, with a mean incidence of $8 \%$ [38].

Another study - of a series of cases involving 1099 patients with confirmed COVID-19 - showed that $13.6 \%$ reported headache and $14.9 \%$ had myalgia [39]. Headache is believed to be secondary to hypoxia induced by decreased blood flow in the vascular bed of the brain and the body's response to inflammatory mediators and cytokines [40].

All respiratory viruses, of which SARS-CoV-2 is one, are known to be able to induce headache. In fact, headache is a generally recognized symptom of systemic viral infection in the International Classification of Headache. The exact mechanisms producing headache associated with this type of infection remain incompletely studied, though it has been shown that the possible causes are linked with fever and exogenous or endogenous pyrogens, the direct actions of microorganisms themselves, and activation of various immunoinflammatory mediators [40]. We also cannot avoid considering the fact that primary forms of headache are also clinically transformed by the new coronavirus infection. The pandemic situation will undoubtedly impose its limitations on standard treatment protocols for both pathophysiological and organizational-social reasons. This will undoubtedly prompt further studies of this question.

Conclusions. Thus, the spectrum of neurological impairments arising in infections with SARS-CoV-2 is extremely wide and diverse, because of the multiple mechanisms of damage to the systems and organs in this infection. Studies of these mechanisms and means for restoring the CNS after SARS-CoV-2 disease constitute an immediate task for applied and theoretical neurology.

The authors declare no conflicts of interests.

\section{REFERENCES}

1. H. R. Niazkar, B. Zibaee, A. Nasimi, and N. Bahri, "The neurological manifestations of COVID-19: a review article," Neurol.Sci., 41, No. 7, 1667-1671 (2020), https://doi.org/10.1007/s10072-020-04486-3. 
2. I. Ahmad and F. A. Rathore, "Neurological manifestations and complications of COVID-19: a literature review," J. Clin. Neurosci., 77, 8-12 (2020), https://doi.org/10.1016/j.jocn.2020.05.017.

3. World Health Organization, Coronavirus Disease (COVID-19) Situation Report - 160, Data as Received by WHO from National Authorities by 10:00 CEST, June 28, 2020, https://www.who.int/ docs/default-source/coronaviruse/situation-reports/20200628-covid19-sitrep-160.pdf?sfvrsn=2fe1c658_2, acc. Aug. 27, 2020.

4. R. Bridwell, B. Long, and M. Gottlieb, "Neurologic complications of COVID-19," Am. J. Emerg. Med., 38, No. 7, 1549.e3-1549.e7 (2020), https://doi.org/0.1016/j.ajem.2020.05.024.

5. H. Fan, X. Tang, Y. Song, et al., "Influence of COVID-19 on cerebrovascular disease and its possible mechanism," Neuropsychiatr. Dis. Treat., 16, 1359-1367 (2020), https://doi.org/10.2147/NDT.S251173.

6. G. Tsivgoulis, L. Palaiodimou, A. H. Katsanos, et al., "Neurological manifestations and implications of COVID-19 pandemic," Ther. Adv. Neurol. Disord., 13, 1756286420932036 (2020), https://doi. org $/ 10.1177 / 1756286420932036$.

7. D. Wang, B. Hu, C. Hu, et al., "Clinical characteristics of 138 hospitalized patients with 2019 novel coronavirus-infected pneumonia in Wuhan, China," JAMA, 323, No. 11, 1061-1069 (2020), https://doi. org/10.1001/jama.2020.1585.

8. J. Wang, N. Hajizadeh, E. E. Moore, et al., "Tissue plasminogen activator (tPA) treatment for COVID-19 associated acute respiratory distress syndrome (ARDS), a case series," J. Thromb. Haemost., 18, No. 7, 1752-1755 (2020), https://doi.org/10.1111/jth.14828.

9. N. Tang, H. Bai, X. Chen, et al., "Anticoagulant treatment is associated with decreased mortality in severe coronavirus disease 2019 patients with coagulopathy," J. Thromb. Haemost., 18, No. 5, 1094 1099 (2020), https://doi.org/10.1111/jth.14817.

10. Y.C. Li, W.Z. Bai, and T. Hashikawa, "The neuroinvasive potential of SARS-CoV2 may play a role in the respiratory failure of COVID-19 patients," J. Med. Virol., 92, No. 6, 552-555 (2020), https://doi.org/10.1002/jmv.25728.

11. H. Cheng, Y. Wang, and G. Q. Wang, "Organ-protective effect of angiotensin-converting enzyme 2 and its effect on the prognosis of COVID-19," J. Med. Virol., 92, No. 7, 726-730 (2020), https://doi. org/10.1002/jmv.2578.

12. L. Steardo, L. Steardo, Jr., R.Zorec, and A. Verkhratsky, "Neuroinfection may contribute to pathophysiology and clinical manifestations of COVID-19," Acta Physiol., 229, No. 3, e13473 (2020), https:// doi.org/10.1111/apha.13473.

13. H. C. Yashavantha Rao and C. Jayabaskaran, "The emergence of a novel coronavirus (SARS-CoV-2) disease and their neuroinvasive propensity may affect in COVID-19 patients," J. Med. Virol., 92, No. 7, 786-790 (2020), https://doi.org/10.1002/jmv.25918.

14. M. Sheraton, N. Deo, R. Kashyap, and S. Surani, "A review of neurological complications of COVID-19," Cureus, 12, No. 5, e8192 (2020), https://doi.org/10.7759/cureus.8192.

15. H. Tu, S. Tu, S. Gao, et al., "The epidemiological and clinical features of COVID-19 and lessons from this global infectious public health event," J. Infect., 81, No. 1, 1-9 (2020), https://doi.org/10. 1016/j.jinf.2020.04.011.

16. K. Tveito, "Cytokine storms in COVID-19 cases," Tidsskr. Nor. Laegeforen, 23, 140 (2020), https://doi.org/10.4045/tidsskr.20.0239.

17. A. Filatov, P. Sharma, and F. Hindi, "Neurological complications of coronavirus disease (COVID-19, encephalopathy," Cureus, 12, No. 3, e7352 (2020), https://doi.org/10.7759/cureus.7352.

18. A.J.Rodriguez-Morales, J.A.Cardona-Ospina,E. Gutiérrez-Ocampo, et al., "Clinical, laboratory and imaging features of COVID-19: a systematic review and meta-analysis," Travel Med. Infect. Dis., 34, 101623 (2020), https://doi.org/10.1016/j.tmaid.2020.101623.

19. L. Mao, H. Jin, M. Wang, et al., "Neurologic manifestations of hospitalized patients with coronavirus disease 2019 in Wuhan, China," JAMA Neurol., 77, No. 6, 1-9 (2020), https://doi.org/10.1001/ jamaneurol.2020.1127.
20. T. Scullen, J. Keen, M. Mathkour, et al., "Coronavirus 2019 (COVID-19)-Associated encephalopathies and cerebrovascular disease: The New Orleans experience," World Neurosurg., 141, e437e446 (2020), https://doi.org/10.1016/j.wneu.2020.05.192.

21. A. O. Correia, P. W. G. Feitosa, J. L. D. S. Moreira, et al., "Neurological manifestations of COVID-19 and other coronaviruses: a systematic review," Neurol. Psychiatry Brain Res., 37, 27-32 (2020), https://doi.org/10.1016/j.npbr.2020.05.008.

22. A. Azhideh, "COVID-19 neurological manifestations," Int. Clin. Neurosci. J., 7, No. 2, 54 (2020), https://doi.org/10.34172/icnj.2020.01.

23. S. T. Reddy, T. Garg, C. Shah, et al., "Cerebrovascular disease in patients with COVID-19: a review of the literature and case series," Case Rep. Neurol., 12, No. 2, 199-209 (2020), https://doi.org/10. $1159 / 000508958$

24. Y. Wu, X. Xu, Z. Chen, et al., "Nervous system involvement after infection with COVID-19 and other coronaviruses," Brain Behav. Immun.,87, 18-22 (2020), https://doi.org/10.1016/j.bbi.2020.03.031.

25. N. Poyiadji, G. Shahin, D. Noujaim, et al., "COVID-19-associated acute hemorrhagic necrotizing encephalopathy: CT and MRI features," Radiology, 296, No. 2, 119-120 (2020), https://doi.org/10. 1148/radiol.2020201187.

26. P. Xiang, X. M. Xu, L. L. Gao, et al., "First case of 2019 novel coronavirus disease with encephalitis," ChinaXiv, T202003.00015 (2020).

27. T. Moriguchi, N. Harii, J. Goto, et al., "A first case of meningitis/ encephalitis associated with SARS-Coronavirus-2," Int. J. Infect. Dis., 94, 55-58 (2020), https://doi.org/10.1016/j.ijid.2020.03.062.

28. R. Brüggemann, H. Gietema, B. Jallah, et al., "Arterial and venous thromboembolic disease in a patient with COVID-19: a case report," Thromb. Res., 191, 153-155 (2020), https://doi.org/10.1016/j. thromres.2020.04.046.

29. T. Chen, D. Wu, H. Chen, et al., "Clinical characteristics of 113 deceased patients with coronavirus disease 2019: retrospective study," $B M J, 368, \mathrm{~m} 1091$ (2020), https://doi.org/10.1136/bmj.m1091.

30. R. Bridwell, B. Long, and M. Gottlieb, "Neurologic complications of COVID-19," Am. J. Emerg. Med., 38, No. 7, 1549.e3-1549.e7 (2020), https://doi.org/10.1016/j.ajem.2020.05.024.

31. A. Filatov, P. Sharma, F. Hindi, and P. S. Espinosa, "Neurological complications of coronavirus disease (COVID-19): encephalopathy," Cureus, 12, No. 3, e7352 (2020), https://doi.org/10.7759/ cureus.7352.

32. M. Benger, O. Williams, J. Siddiqui, and L. Sztriha, "Intracerebral haemorrhage and COVID-19: clinical characteristics from a case series," Brain Behav. Immun., 88, 940-944 (2020), https://doi.org/10. 1016/j.bbi.2020.06.005

33. A. Sharifi-Razavi, N. Karimi, and N. Rouhani, "COVID-19 and intracerebral haemorrhage: causative or coincidental," New Microbes New Infect., 35, 100669 (2020), https://doi.org/10.1016/j.nmni.2020. 100669.

34. J. Sotoca and Y. Rodríguez-Álvarez, "COVID-19-associated acute necrotizing myelitis," Neurol. Neuroimmunol. Neuroinflamm., 7, No.5,e803(2020),https://doi.org/10.1212/NXI.0000000000000803.

35. R. AlKetbi, D. AlNuaimi, M. AlMulla, et al., "Acute myelitis as a neurological complication of Covid-19: a case report and MRI findings," Radiol. Case Rep., 15, No. 9, 1591-1595 (2020), https://doi. org/10.1016/j.radcr.2020.06.001.

36. M. Munz, S. Wessendorf, G. Koretsis, et al., "Acute transverse myelitis after COVID-19 pneumonia," J. Neurol., 267, No. 8, 21962197 (2020), https://doi.org/10.1007/s00415-020-09934-w.

37. N. Poyiadji, P. Cormier, P. Y. Patel, et al., "Acute pulmonary embolism and COVID-19," Radiology, 297, No. 3, 335-338 (2020), https://doi.org/10.1148/radiol.2020201955.

38. N. Tang, H. Bai, X. Chen, et al., "Anticoagulant treatment is associated with decreased mortality in severe coronavirus disease 2019 patients with coagulopathy," J. Thromb. Haemost., 18, No. 5, 1094 1099 (2020), https://doi.org/10.1111/jth.14817. 
39. M. Desforges, A. Le Coupanec, P. Dubeau, et al., "Human coronaviruses and other respiratory Viruses: underestimated opportunistic pathogens of the central nervous system," Viruses, 12, No. 1, 14 (2019), https://doi.org/10.3390/v12010014.
40. F. Liu, L. Li, M. Xu, et al., "Prognostic value of interleukin-6, C-reactive protein, and procalcitonin in patients with COVID-19," J. Clin. Virol., 127, 104370 (2020), https://doi.org/10.1016/j.jcv. 2020.104370 . 